\title{
La Universidad de Chile no es neutral con respecto a la democracia ambiental y el desarrollo sostenible
}

\author{
University of Chile is not neutral with respect to environmental \\ democracy and sustainable development
}

\section{Valentina Durán Medina (iD}

Directora

La Universidad de Chile reconoce, entre sus valores más preciados, el pluralismo, el respeto a la diversidad, el diálogo, la tolerancia y las libertades de conciencia, de cátedra, pensamiento y expresión. Al mismo tiempo, como institución de educación superior de carácter estatal y parte de un Estado republicano democrático, la universidad se define desde un compromiso esencial con el interés general del país, la igualdad social y de género, el pluralismo intelectual, moral y político, lo que incluye la promoción de los derechos humanos y su respeto irrestricto en todas sus dimensiones. ${ }^{1}$

En esta ocasión queremos rescatar cómo de acuerdo con nuestra misión, visión y con las reglas de derecho público que nos rigen, la Universidad de Chile y quienes la integramos no estamos llamados a ser neutrales ante los desafíos de la democracia ambiental y del desarrollo sostenible.

En primer lugar, en tanto universidad del Estado, nos rige el deber constitucional de «velar por el ejercicio del derecho a vivir en un medio ambiente sano y tutelar la preservación de la naturaleza» establecido en el artículo 19, número 8, de la Constitución, como correlato del derecho a vivir en un medio ambiente libre de contaminación.

Luego, la Ley 21.094 sobre universidades estatales, en su artículo 1, integra dentro de la definición de las universidades del Estado la finalidad de contribuir al fortalecimiento de la democracia y al desarrollo sustentable e integral del país. ${ }^{2}$

1. Para más información sobre la misión y la visión de la Universidad de Chile, los enlaces a sus Estatutos, el Proyecto de Desarrollo Institucional y otros documentos, véase https://uchile.cl/u39635C.

2. La Ley 21.094 define a las universidades estatales como «instituciones de educación superior de carácter estatal, creadas por ley para el cumplimiento de las funciones de docencia, investigación, creación 
En la misma línea, en su artículo 3, los Estatutos de la Universidad de Chile establecen que la casa de estudios postula el desarrollo sostenible del país, ya que, «en cumplimiento de su labor, la universidad responde a los requerimientos de la nación, constituyéndose como reserva intelectual caracterizada por una conciencia social, crítica y éticamente responsable». Además, reconoce, como parte de su misión, la atención de los problemas y necesidades del país, al mismo tiempo que agrega que:

Con ese fin se obliga al más completo conocimiento de la realidad nacional y a su desarrollo por medio de la investigación y la creación; postula el desarrollo integral, equilibrado y sostenible del país, aportando a la solución de sus problemas desde la perspectiva universitaria, y propende al bien común y a la formación de una ciudadanía inspirada en valores democráticos, procurando el resguardo y el enriquecimiento del acervo cultural nacional y universal. ${ }^{3}$

Por otra parte, la visión de futuro de la Universidad de Chile, contenida en su Plan de Desarrollo Institucional 2017-2026, establece que busca responder «a los retos que derivan de las transformaciones que tanto globalmente como localmente han ocurrido y seguirán ocurriendo en la sociedad, el conocimiento, la tecnología, la cultura y el medio ambiente». Además, se propone:

Avanzar en la vinculación de la labor académica y de la comunidad universitaria en el marco de los desafíos y necesidades de nuestra sociedad, de la región latinoamericana, del concierto internacional y del medio ambiente, con el fin de cumplir con el compromiso público de la Universidad de Chile con el desarrollo sustentable del país y la sociedad. ${ }^{4}$

Entonces, existe un compromiso público con el desarrollo sustentable del país y la sociedad, así como con los objetivos relacionados con la conexión de la función académica con sus necesidades. Asimismo, la universidad se esfuerza por desarrollar una mirada transdisciplinaria para el diseño de soluciones de los problemas estratégicos del país, como el que representa el cambio climático, que no puede sino abordarse desde la transdisciplina.

artística, innovación, extensión, vinculación con el medio y el territorio, con la finalidad de contribuir al fortalecimiento de la democracia, al desarrollo sustentable e integral del país, y al progreso de la sociedad en las diversas áreas del conocimiento y dominios de la cultura».

3. Decreto con Fuerza de Ley 3 del 10 de marzo de 2006 del Ministerio de Educación, D.O. 2 de octubre de 2007, que fija texto refundido, coordinado y sistematizado del Decreto con Fuerza de Ley 153 de 1981, que establece los Estatutos de la Universidad de Chile. Para más información, véase https://uchile. $\mathrm{cl} / \mathrm{u} 58046$. El destacado es nuestro.

4. Para más información, véase «Plan de Desarrollo Institucional 2017-2016», aprobado por el Senado Universitario de la Universidad de Chile, Decreto Universitario o031884/2018, disponible en https:// uchile.cl/u30784. El destacado es nuestro. 
Este compromiso se ha expresado con especial fuerza en los últimos dos años, con la puesta en marcha más activa de la Política de Sustentabilidad Universitaria aprobada en 2012 por el Senado Universitario, cuyo decreto fue emitido en 2016. ${ }^{5}$ Esta Política aborda los desafíos y acciones necesarias en la línea de la sustentabilidad en las cuatro áreas del quehacer universitario: docencia, extensión, investigación y gestión.

En aplicación de la mencionada Política de Sustentabilidad, en la Facultad de Derecho, en agosto en 2019, por Resolución 1036 del Decano Pablo Ruiz-Tagle, se creó el Comité Local de Unidad Sustentable, integrado por representantes académicos, funcionarios y estudiantiles, como órgano asesor de las labores del vicedecanato en materias de desarrollo sustentable. Presidido por la vicedecana Renée Rivero, la secretaría técnica del Comité está alojada en el Centro de Derecho Ambiental.

En 2019, con ocasión de la presidencia de Chile de la COP 25 de Cambio Climático, el rector Ennio Vivaldi convocó a un grupo asesor ad hoc para preparar a la universidad para este encuentro global, y hacer visible y más ambiciosa su acción climática.

Después de que en agosto de 2019 la Universidad de Chile se uniera a 150 universidades en un llamado global a declarar «emergencia climática», ${ }^{6}$ en su octava sesión, en 2020, el Consejo Universitario respaldó unánimemente el «Manifiesto de la Universidad de Chile: El desafío histórico de la humanidad frente al cambio climático»,7 que fue propuesto por el rector en junio después del trabajo de este grupo ad hoc, en el que participaron activamente representantes del Centro de Derecho Ambiental junto con académicas de distintas unidades. Digo bien «académicas», ya que el mismo rector destacó públicamente el especial compromiso de las mujeres de la universidad con el cometido. El Manifiesto fue presentado a la comunidad por el rector el 2 de julio de 2020 en la inauguración de las IX Jornadas de Derecho Ambiental, con las que el Centro de Derecho Ambiental celebró sus veinte años. ${ }^{8}$

En gestión, el documento contiene el compromiso de la universidad de ser carbono neutral al 2050, comprometiéndose a revisar este término hacia una mayor ambición, además de otras medidas, como la generación de un catastro del Patrimonio Natural que alberga la universidad, el fortalecimiento del Comité de Sustentabilidad

5. Para más información, véase «Política de Sustentabilidad Universitaria», aprobada por unanimidad en el Senado Universitario de la Universidad de Chile el 12 de julio de 2012, vigente mediante Resolución de Rectoría 1424/2016, disponible en https://uchile.cl/u165455.

6. Para más información, véase «U. de Chile se une al llamado de más de 150 universidades para declarar emergencia climática global», Universidad de Chile,2 de septiembre de 2019, disponible en https:// uchile.cl/u157332.

7. Para más información, véase «Manifiesto de la Universidad de Chile: El desafío histórico de la humanidad frente al cambio climático», disponible bit.ly/3aWGT6g.

8. El discurso inaugural de las IX Jornadas de Derecho Ambiental y otros videos están disponibles en bit.ly/38H2lco. Para más información, véase http://uchile.cl/d165037. 
Universitaria y la creación de una futura Dirección de Sustentabilidad. En cuanto a la docencia, además de la ampliación de la oferta de pre y posgrado se plantea, entre otros, introducir la macrocompetencia de sustentabilidad en el Modelo Educativo expresado en los perfiles de egreso. En investigación, el Manifiesto propone, entre otras medidas, crear incentivos para la formación de redes que trabajen en temáticas vinculadas al medio ambiente, así como generar una instancia de divulgación científica que permita a la sociedad conocer a especialistas y a sus propuestas en desarrollo sostenible. En extensión y formación continua, el texto compromete, entre otros, incorporar indicadores de sustentabilidad que permitan evaluar el desempeño a nivel institucional y tanto reconocer como desarrollar programas de universidad abierta, además de apoyar la integración de prácticas sustentables al interior de los Campus.

Entre sus considerandos, el Manifiesto expresa honrar el llamado transversal al que la Universidad de Chile se sumó en mayo de 2020, para impulsar una «reactivación sostenible» pospandemia, es decir, la adopción de estrategias de recuperación económica, cuyo objetivo esté centrado en la construcción de una sociedad más justa, sostenible, inclusiva y resiliente. ${ }^{9}$

Son muchas las iniciativas, programas, proyectos y unidades académicas que han ido respondiendo a este llamado desde las más diversas disciplinas, saberes y artes con el apoyo del nivel central de la Universidad de Chile, así como de las distintas facultades e institutos. También debemos destacar numerosas iniciativas estudiantiles como la Escuela Ambiental Interdisciplinaria,${ }^{10}$ ganadora de un Premio Azul y nacida al alero del Centro de Derecho Ambiental, y el compromiso de las funcionarias y los funcionarios que se integran activamente en los Comités Locales de Unidad Sustentable conformados en las distintas facultades.

Como hemos expuesto, la Universidad de Chile, por mandato tanto constitucional como legal y no solo conforme a su visión, sino también a su misión institucional, tiene un papel relevante que cumplir en la acción climática y hacia un desarrollo sostenible y de protección de las generaciones futuras.

Siendo el lugar donde todas las verdades se tocan, como afirmó Andrés Bello en su célebre discurso pronunciado en la instalación de la Universidad de Chile el 17 de septiembre de 1843 , ${ }^{11}$ la universidad también es un espacio que reconocemos tanto diverso como abierto a la libertad de cátedra y de opinión. Esta diversidad no obsta a que la universidad pueda y deba tomar posición institucional en las materias que, como la crisis social y ambiental, involucran la violación de derechos humanos y se constituyen como mínimos civilizatorios para asegurar la subsistencia de la humanidad en nuestro planeta y tanto la vigencia de la democracia como del Estado de derecho.

9. Para más información sobre este punto, véase bit.ly/2JvFDLR.

10. Para más información sobre la Escuela Ambiental Interdisciplinaria, véase http://uchile.cl/d171901.

11. El discurso mencionado se encuentra disponible en https://uchile.cl/u4682. 
Por todo lo anterior, afirmo que la Universidad de Chile no es ni debe ser neutral cuando se trata del desarrollo sostenible y de la democracia ambiental.

Me atrevo a situar en este plano el apoyo universitario que ha recibido el Acuerdo de Escazú, que el gobierno de Chile, tristemente, decidió no firmar. Escazú, el primer tratado ambiental de América Latina y el Caribe, busca garantizar la implementación plena y efectiva de los derechos de acceso a la información ambiental, la participación pública en los procesos de toma de decisiones ambientales y el acceso a la justicia en asuntos ambientales en la región, así como la creación y el fortalecimiento de las capacidades y la cooperación, contribuyendo a la protección del derecho de cada persona, de las generaciones presentes y futuras, a vivir en un medio ambiente sano, y al desarrollo sostenible. A la «Declaración del Comité por la Sustentabilidad de la Universidad de Chile con respecto al Acuerdo de Escazú: firma y ratificación del Estado de Chile» de septiembre de 2020, ${ }^{12}$ se suman decenas de acciones estudiantiles y académicas de estudio e impulso a la firma e implementación por Chile de este tratado $y$, recientemente, en la nueva serie de publicaciones del Centro de Derecho Ambiental, el segundo número de \#PerspectivasCDA titulado ¿Por qué Chile debe adherir al Acuerdo de Escazú? ${ }^{13}$

Por último, en este marco destacamos la decisión de la Facultad de Derecho y de nuestro Centro de Derecho Ambiental de ser parte activa de la discusión constitucional, luego del rotundo triunfo de las opciones «Apruebo» $y$ «Convención constituyente» en el plebiscito del 25 de octubre de 2020. Pronto daremos a conocer un documento con ciertos contenidos mínimos, en el marco de las justas diferencias, que desde el Centro de Derecho Ambiental proponemos que sean abordados en la discusión que se avecina.

Cierro estas líneas editoriales con agradecimientos. En primer lugar, quiero agradecer a todo el equipo académico y funcionario del Centro de Derecho Ambiental (CDA), así como a sus ayudantes, por el enorme esfuerzo desplegado en un año tan difícil. ${ }^{14}$ En la convicción de que no somos neutrales y que nos inspiran principios superiores, el equipo siguió investigando, postulando proyectos, realizando docencia de pre y posgrado, contribuyendo a la definición de políticas públicas y debates legislativos, estudiando jurisprudencia, y ofreciendo actividades de extensión de gran calidad, como los talleres constitucionales, ${ }^{15}$ las IX Jornadas de Derecho Ambiental, ${ }^{16}$

12. Para más información, véase «Declaración del Comité por la Sustentabilidad de la Universidad de Chile respecto al Acuerdo de Escazú: firma y ratificación del Estado de Chile», Universidad de Chile, 25 de septiembre de 2020, disponible en https://uchile.cl/u168949.

13. De autoría de Valentina Durán Medina y Constance Nalegach Romero, el texto se encuentra disponible en http://uchile.cl/d166356.

14. Conozca al equipo del CDA en http://uchile.cl/d88594.

15. El video promocional de los talleres constitucionales en línea está disponible en bit.ly/2MlrVwi.

16. Para más información sobre las IX Jornadas de Derecho Ambiental, véase http://uchile.cl/d169147. 
las III Jornadas de Régimen Jurídico de las Aguas ${ }^{17}$ organizadas junto al Centro de Regulación y Competencia RegCom, y el concurrido Ciclo «Ecoderecho, foros virtuales para una constitución del siglo XXI», organizado junto al Centro de Derechos Humanos. ${ }^{18}$

En segundo lugar, agradezco al equipo editorial de la Revista de Derecho Ambiental por su persistente y encomiable trabajo, que nos llevó, luego de un proceso de evaluación de nueve meses, a ser aceptados en Scopus, la prestigiosa base de datos de referencias bibliográficas y citas, de literatura peer review y contenido en línea de calidad, con herramientas para el seguimiento, análisis y visualización de la investigación. Para lograrlo, la Revista tuvo que cumplir estrictos indicadores de calidad, como procurar una revisión ciega por pares, una publicación regular, poseer un mínimo de dos años de historia de publicaciones, altos estándares de ética, negligencia y control del plagio, normas claras sobre derechos de autor, y tanto licencias como integración de expertos reconocidos de la disciplina en su Comité Editorial.

En palabras del Content Selection \& Advisory Board, comité encargado de aceptar las publicaciones a Scopus, «la revista, consistentemente, incluye artículos que son científicamente sólidos y relevantes para una audiencia académica o profesional internacional en este campo», reconociendo la contribución al derecho ambiental de la publicación que actualmente pasa a formar parte de esta base de datos junto con otras revistas de la facultad y de la Universidad de Chile, en su persistente trabajo de orientar su quehacer al avance de la ciencia y el conocimiento.

Como resultado, actualmente la Revista de Derecho Ambiental se encuentra indexada en DOAJ, Latindex, Redib y Scopus, con el apoyo de las autoridades de la Facultad, de la Dirección de Servicios de Información y Bibliotecas, del Programa de Revistas de la Dirección de Investigación de la Facultad de Derecho de la Universidad de Chile, de la Dirección de Servicios de Información y Bibliotecas (SISIB) y de Tipográfica, a quienes también agradecemos sinceramente. ${ }^{19}$

La Revista de Derecho Ambiental — coeditada por Jorge Ossandón y Antonio Pulgar junto al equipo de los ayudantes Débora Quiero, Karla Vargas y Carlos Soto, la consejería del profesor Jorge Aranda, la subdirección de la profesora Pilar Moraga, la colaboración de las profesoras Ximena Insunza y Ana Lya Uriarte y del profesor Sergio Montenegro, y el apoyo de nuestro Comité Editorial que integran los profeso-

17. Para más información, véase «Jornadas del Régimen Jurídico de las Aguas», Universidad de Chile, disponible en bit.ly/34YnxJV.

18. Para información, véase «Ciclo «Ecoderecho»: Un encuentro de miradas en torno a la protección del medio ambiente y la Constitución", Universidad de Chile, 19 de mayo de 2020, disponible en http:// uchile.cl/d163579. Además, los videos del ciclo completo y de todas las actividades de extensión se encuentran disponibles en la lista de reproducción del Centro de Derecho Ambiental en bit.ly/38Jo2Ws.

19. Para más información, véase «Revista de Derecho Ambiental pasa a formar parte de Scopus», Univesidad de Chile, 3 de agosto de 2020, disponible en https://uchile.cl/u165851 . 
res Verónica Delgado, Juan Carlos Ferrada, Iván Hunter Ampuero, Alberto Olivares, Lina Muñoz-Ávila, y Carina Costa de Oliveira, la secretaría de Daniela Vargas y bajo la dirección de quien suscribe- continúa fortaleciéndose como un espacio de exposición y análisis en el plano académico del derecho ambiental. Nada de esto sería posible sin el esfuerzo de los autores y autoras que confían en nuestra revista, así como de nuestros pares evaluadores, cuyas contribuciones anónimas agradecemos.

Esperamos que este número, compuesto por diez artículos de investigación, todos muy diversos y que abordan temas de interés nacional e internacional para el desarrollo del derecho ambiental, sea de agrado para nuestros lectores.

\section{Sobre la autora}

Valentina Durán Medina es abogada por la Universidad de Chile, máster en Derecho Ambiental por las universidades de París I Panthéon-Sorbonne y París II Panthéon-Assas. Además, es profesora asociada de la Facultad de Derecho de la Universidad de Chile, donde dirige el Centro de Derecho Ambiental. También es docente de los Departamentos de Enseñanza Clínica del Derecho y de Derecho Económico. Pertenece al claustro del magíster de la misma facultad, dirige la Revista de Derecho Ambiental y el diplomado de Derecho Ambiental e Instrumentos de Gestión. Su correo electrónico es vduran@derecho.uchile.cl. (D) http://orcid.org/0000-0002-9347-1017. 
La Revista de Derecho Ambiental, del Centro de Derecho Ambiental de la Facultad de Derecho de la Universidad de Chile, es un espacio de exposición y análisis en el plano académico del derecho ambiental. Su contenido se presenta a través de doctrina, jurisprudencia y recensiones, y aborda diversas materias relacionadas con la gestión, institucionalidad y herramientas de protección ambiental y desarrollo sustentable. Se presentan artículos de diferentes autores y autoras en los que se analizan y abordan casos y temas jurídico-ambientales de creciente interés y actualidad.

\author{
DIRECTORA \\ Valentina Durán Medina \\ EDITORES \\ Jorge Ossandón Rosales \\ y Antonio Pulgar Martínez \\ SITIO WEB \\ revistaderechoambiental.uchile.cl \\ CORREO ELECTRÓNICO \\ revistada@derecho.uchile.cl \\ LICENCIA DE ESTE ARTÍ́CULO \\ Creative Commons Atribución Compartir Igual 4.o Internacional
}

La edición de textos, el diseño editorial

y la conversión a formatos electrónicos de este artículo

estuvieron a cargo de Tipográfica

(www.tipografica.io) 